\title{
Neural Networks approach to event reconstruction for the GAPS experiment
}

\author{
N. Marcelli ${ }^{a, b, *}$ on behalf of the GAPS Collaboration \\ (a complete list of authors can be found at the end of the proceedings) \\ "University of Rome "Tor Vergata", \\ Department of Physics, I-00133 Rome, ItalyInstitution \\ ' INFN, Sezione di Rome "Tor Vergata", \\ I-00133 Rome, Italy \\ E-mail: nadir.marcelli@roma2.infn.it
}

The General Antiparticle Spectrometer (GAPS) is a balloon-borne experiment, scheduled for a first flight in the austral summer 2022. It is designed to measure low energy $(<0.25 \mathrm{GeV} / n)$ cosmic antinuclei. A particular focus is on antideuterons, which are predicted to have an ultra-low astrophysical background as compared to signals from dark matter annihilation or decay in the Galactic halo. GAPS uses a novel technique for particle identification based on the formation and decay of exotic atoms. To achieve sufficient rejection power for particle identification, an accurate determination of several fundamental quantities is needed. The precise reconstruction of the energy deposition pattern on the primary track is a particularly intricate problem and we developed a strategy devised to solve this using modern machine learning techniques. In the future, this approach can also be used for particle identification. Here, we present preliminary results of these efforts obtained from simulations.

$37^{\text {th }}$ International Cosmic Ray Conference (ICRC 2021)

July 12th - 23rd, 2021

Online - Berlin, Germany

\footnotetext{
${ }^{*}$ Presenter
} 


\section{Introduction}

Dark matter (DM) constitutes $\sim 26 \%$ of energy budget of the Universe, about five times more abundant than baryonic matter, but its fundamental nature is still unknown. DM evidences came only by gravitational observations, indicating that if it exists, it must barely interact with ordinary baryonic matter and radiation. Particularly appealing candidates for DM particles are electrically neutral and weakly interacting massive particles (WIMPs) with a mass in the range between $10 \mathrm{GeV}$ and a few $\mathrm{TeV}$ [1]. Indirect searches for DM aim at detecting the signatures of possible annihilations or decays of DM particles in the fluxes of cosmic rays (CRs). A key point of these searches is to look for channels and ranges of energy where it is possible to extrapolate a DM signal from ordinary astrophysical background. This is the basic reason why searches for charged particles focus on fluxes of antiparticles (positrons, antiprotons, antideuterons), much less abundant in the Universe than their corresponding particles [2].

In particular, antideuterons could lead to a breakthrough discovery of DM [3, 4]. Due to the kinematics of the antinuclei formation, the possible flux of DM-produced cosmic antideuterons below few $\mathrm{GeV} / n$ is predicted to be several orders of magnitude above the expected flux of antideuterons from astrophysical origin (i.e., from secondary interactions of cosmic rays). The General Antiparticle Spectrometer (GAPS) is specifically designed for low energy $(<0.25 \mathrm{GeV} / n)$ cosmic antinuclei detection [5-8].

\section{The GAPS detector}

The GAPS experiment is a balloon-borne detector, scheduled for the first of three flights in Antarctica during the austral summer 2022-2023. The in-situ low geomagnetic cutoff is ideal for the detection of low energy cosmic particles. Fig. 1 shows a schematic view of the GAPS apparatus consisting of a Time-of-Flight (ToF) and tracking systems [9].

The ToF system consists of $\sim 160$ plastic scintillator paddles [10] and is arranged in an outer Tof system and an inner Tof system (see Fig. 1 right). The outer ToF consists of an umbrella of scintillator oriented horizontally and a cortina of four walls of scintillator oriented vertically. The inner ToF is a cube of scintillator, consisting of four sides, a top and a bottom. Each plastic scintillator paddle is $6.35 \mathrm{~mm}$ thick and $16 \mathrm{~cm}$ wide. The umbrella consists of $1.8 \mathrm{~m}$ and $1.51 \mathrm{~m}$ length paddles, whereas the cortina and the cube use $1.72 \mathrm{~m}$ and $1.51 \mathrm{~m}, 1.8 \mathrm{~m}$ and $1.56 \mathrm{~m}$ lengths respectively. The ToF system measures the time information necessary to reconstruct velocity of particles and the ionization energy losses $\mathrm{d} E / \mathrm{d} x$ of particles. The ToF also provides the overall trigger for GAPS.

The core of the apparatus is a tracking system made of ten planes of $12 \times 12$ cylindrical $\mathrm{Si}(\mathrm{Li})$ detectors each [11-13]. On each supporting aluminum plane, the $\mathrm{Si}(\mathrm{Li})$ cylinders are arranged in a $6 \times 6$ array of modules, each with four $\mathrm{Si}(\mathrm{Li})$ detectors read-out by a dedicated ASIC [14]. The $\mathrm{Si}(\mathrm{Li})$ detectors have a diameter of $\sim 10 \mathrm{~cm}$, are $\sim 2.5 \mathrm{~cm}$ thick and are segmented into eight strips of equal area. Finally, the tracking system is kept at his operational temperature of $\sim-40^{\circ} \mathrm{C}$.

GAPS uses a novel technique for antinuclei detection, based on formation and decay of exotic atoms. The incoming primary antinucleus slows down due to ionization energy losses through the materials of the apparatus and can form an exotic atom by replacing a shell electron in a silicon 


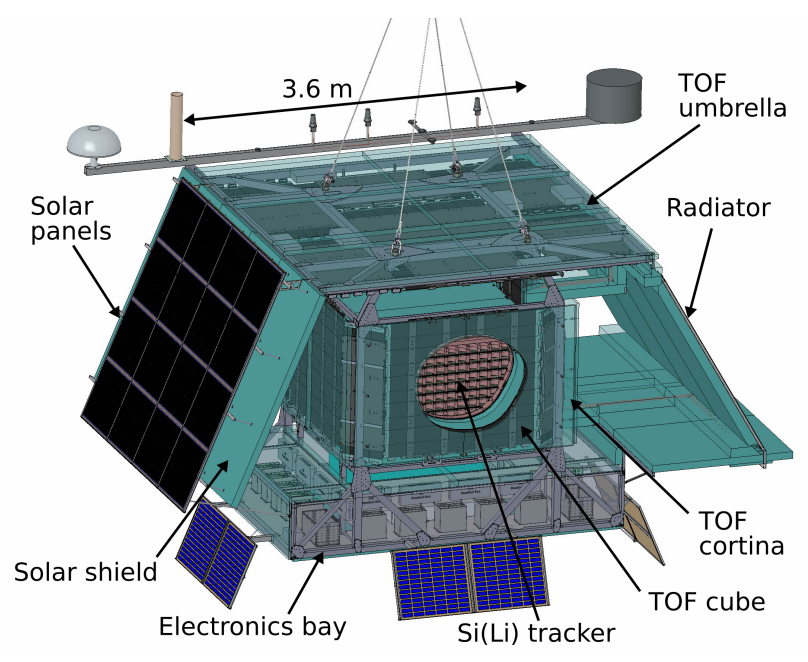

Figure 1: A schematic view of the GAPS detector.

or aluminum atom. During the de-excitation process, the exotic atom emits characteristic X-rays with energies determined by atomic transitions. At the end, the primary particle annihilates with the target nucleus producing secondary particles, mainly pions and protons from a common vertex. The measurements of time of flight, ionization losses $\mathrm{d} E / \mathrm{d} x, \mathrm{X}$-ray energies together with the reconstructed topology of the "annihilation star", allow to clearly identify antinuclei.

\section{Energy deposition reconstruction}

In order to reconstruct the antinucleus annihilation topology, a custom algorithm was developed. First, the primary track is identified, followed by the secondary tracks associated with the primary track. Finally, the annihilation vertex is defined as the point which minimize the distance between primary and secondary reconstructed tracks. More details can be found in [15, 16]. The reconstruction algorithm was developed using the GAPS simulation software, which reproduces the instrument geometry and materials using the Geant4 toolkit [17]. Moreover, a digitization process was applied on the simulated data to mimic a realistic instrument response for time, energy, and position measurements.

The reconstruction algorithm provides several quantities related to the primary, one of the most relevant for particle identification is the ionization energy deposition. Since the digitization affects the energy reconstruction, it is important to compare the primary reconstructed energy with its Monte-Carlo-truth (MC) value. When comparing the MC total energy deposited in the detectors via ionization by the primary particle with the total digitized energy in the same detector, in some cases an excess is observed with respect to the digitized information.

This excess results from a combination of two effects:

- The primary antinucleus annihilates in a detector and the produced secondary particles release energy in the same volume

- Secondary particles can cross a volume already crossed by the primary in a time scale smaller than the integration time of the detector 

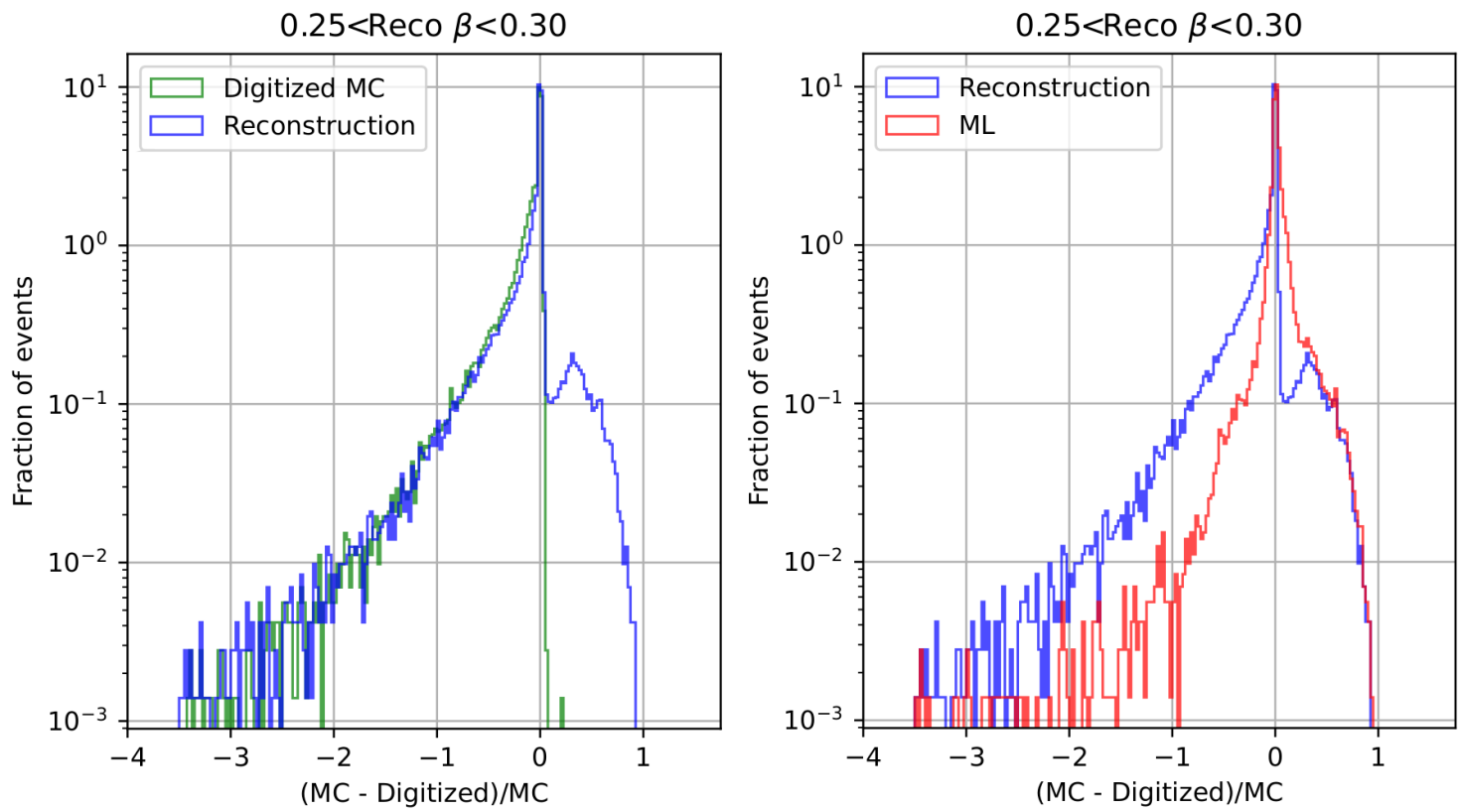

Figure 2: Left panel: residuals between total MC primary energy deposition and the sum of all digitized deposits associated with the primary according to $\mathrm{MC}$ (green histogram) and with the reconstruction algorithm (blue histogram). Right panel: residuals between total MC primary energy deposition and the sum of digitized deposits associated with the primary according to reconstruction algorithm before and after applying the ML correction (blue and red histograms respectively). Both panels refer to the interval of reconstructed primary $\beta$ between 0.25 and 0.30 .

The effect was investigated using the Monte Carlo information. The $\mathrm{MC}$ total energy deposited via ionization by the slowing-down primary was compared in $\beta$ ( $\beta=v / c, v$ velocity of the primary, $c$ speed of light) intervals with the total digitized energy in all detectors crossed by the primary according to the $\mathrm{MC}$ and the reconstruction algorithm. The green and blue histograms in Fig. 2 (left) show the residuals for one of the $\beta$ intervals. Both histograms peak at zero, however, a clear asymmetry can be observed with a long tail for negative values and, for the blue histogram, an additional distribution peaking around 0.5 for positive values. The negative tail, represents the energy excess, while the feature around 0.5 is due to missing hits on the reconstructed primary track.

In order to reduce the effect of energy excess in the digitized total primary energy a Neural Network (NN) was developed. A NN is usually made of a sequence of simple data transformations (layers) and these data transformations are learned by exposure to examples [18]. Each layer applies a few simple tensor operations to the input data, serving its output to the next layer. The specification of what a layer does to its input data is stored in the layer's weights, which are a set of numbers. During the NN training phase, the weights are updated at each iteration (epoch) to reduce the distance between predictions and true values. The function which defines a distance score is named loss function.

The used NN architecture is a type of Recurrent Neural Network (RNN) known as Long-Short Term Memory (LSTM) [19], it can process a sequence of data extracting information not only by the single elements, but also by their order. The input to the $\mathrm{NN}$ is a time series corresponding to 

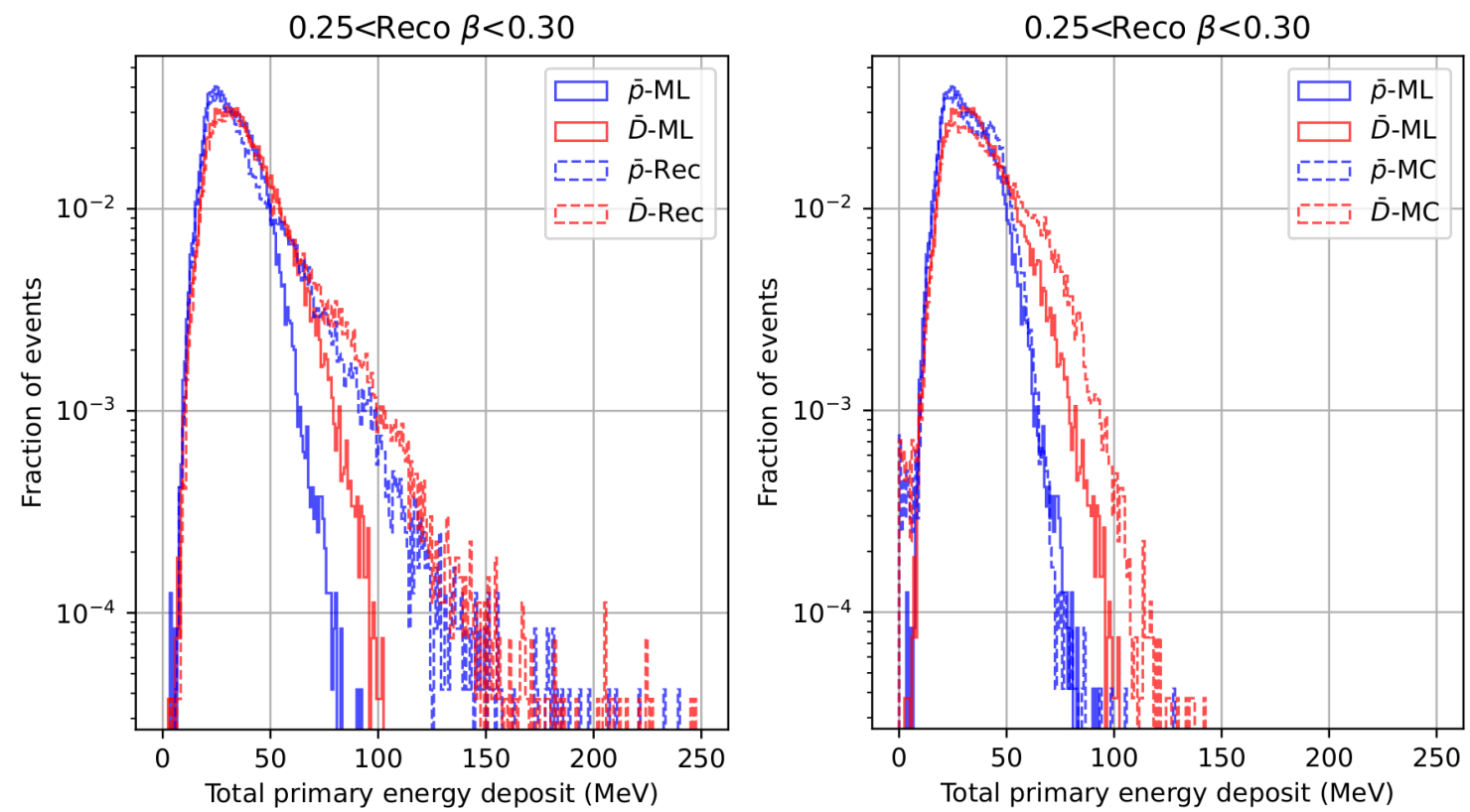

Figure 3: Left panel: total energy distribution obtained as the sum of all digitized (dashed histogram) and ML corrected (solid histogram) primary energy deposits. Right panel: comparison between the total primary energy distributions obtained summing all MC (dashed histogram) and ML corrected (solid histogram) primary deposits. Both panels refer to the interval of reconstructed primary $\beta$ between 0.25 and 0.30 . Blue histograms are for antiprotons $(\bar{p})$ and red histograms are for antideuterons $(\bar{D})$.

the sequence of digitized energy deposits associated with the primary (according to reconstruction algorithm) and returns as output target the same deposits according to real MC primary energy deposits.

The choice of a LSTM was driven mainly by two reasons

- It can easily manage variable length input (and output) sequences: the number of detectors crossed by the primary varies with velocity and arrival direction of the primary particle.

- It can extract information by the order of the input sequence following the slow down profile due to ionization energy loss of the primary

The best architecture configuration for this work was found to be two LSTM layers with 128 and 64 channels, with the last one-dimensional linear output LSTM layer. The loss function minimized during training is a mean square error, defined as the mean squared difference between $\mathrm{MC}$ and ML corrected energy depositions. A train and test dataset was selected requiring a reconstructed annihilation vertex contained inside the cube volume. The $\mathrm{NN}$ was trained for 70 epochs on a sample of 300000 antiprotons and antideuterons for a total of 600000 events. The mean absolute error (MAE), defined as the mean absolute difference between the MC and ML corrected energy depositions, was computed separately for antiproton and antideuteron test samples. The obtained results with this configuration are $\bar{p}_{M A E}=0.63 \mathrm{MeV}$ and $\bar{D}_{M A E}=0.69 \mathrm{MeV}$.

Fig. 2 (right panel) shows the residuals between total MC primary energy deposition and the sum of digitized deposits associated with the primary according to reconstruction algorithm before 
and after applying the Machine Learning (ML) correction (blue and red histograms respectively). A clear reduction of the energy excess can be observed with the ML output.

Fig. 3 shows the distributions of the total primary energy obtained summing all the energy depositions associated with the primary according to reconstruction, ML corrections, and MC. Differences between the two species increase after applying the ML correction (solid histograms). This is one of the quantities that will be used for antinuclei identification [20]. In the right panel of Fig. 3 the dashed histograms represent the sum of MC primary energy depositions, which can be interpreted as the best possible information achievable. From that figure it can be seen that the NN output slightly underestimates the antideuteron total MC primary energy. The remaining difference is due to occasional wrong association of detector hits to the primary track by the reconstruction algorithm. Antideuteron events have on average a greater number of registered hits with respect to antiprotons, which increase the chance of missing hits on the reconstructed primary track.

\section{Conclusions}

To achieve sufficient rejection power for particle identification, an accurate determination of several physical quantities is needed. A precise reconstruction of the energy deposition profile on the primary track is very important. In this work, we present an approach to treat the accurate energy deposition reconstruction using modern machine learning techniques. The results show that the RNN can reproduce the MC simulated data from the digitized signal relative to the primary track with a low MAE $(\sim 0.66 \mathrm{MeV})$. The total energy deposition of the primary, corrected with the ML tool, has the potential to significantly benefit the antiproton and antideuteron identification analysis.

\section{Acknowledgments}

This work is supported in the U.S. by NASA APRA grants (NNX17AB44G, NNX17AB45G, NNX17AB46G, and NNX17AB47G) and in Japan by JAXA/ISAS Small Science Program FY2017. P. von Doetinchem received support from the National Science Foundation under award PHY1551980 . H. Fuke is supported by JSPS KAKENHI grants ( JP17H01136 and JP19H05198 ) and Mitsubishi Foundation Research Grant 2019-10038. K. Perez and M. Xiao are supported by Heising-Simons award 2018-0766. F. Rogers is supported through the National Science Foundation Graduate Research Fellowship under Grant No. 1122374 . Y. Shimizu receives support from JSPS KAKENHI grant JP20K04002 and Sumitomo Foundation Grant No. 180322. This work is supported in Italy by Istituto Nazionale di Fisica Nucleare (INFN) and by the Italian Space Agency through the ASI INFN agreement no. 2018-28-HH.0: "Partecipazione italiana al GAPS - General AntiParticle Spectrometer". The technical support and advanced computing resources from the University of Hawaii Information Technology Services - Cyberinfrastructure are gratefully acknowledged. This research was done using resources provided by the Open Science Grid [21, 22], which is supported by the National Science Foundation award \#2030508. 


\section{References}

[1] M. Boezio et al. PAMELA and indirect dark matter searches. New Journal of Physics, 11(10):105023, Oct 2009.

[2] M. Cirelli. Status of Indirect (and Direct) Dark Matter searches. PoS, ICRC2015:014, 2016.

[3] F. Donato et al. Antideuterons as a signature of supersymmetric dark matter. Phys. Rev. D, 62:043003, Jul 2000.

[4] P. von Doetinchem et al. Cosmic-ray antinuclei as messengers of new physics: status and outlook for the new decade. Journal of Cosmology and Astroparticle Physics, 2020(08):035035, aug 2020 .

[5] M. Xiao et al. In search of cosmic-ray antinuclei from dark matter with the GAPS experiment. This conference Proceedings of Science, 2021.

[6] C. J. Hailey. An indirect search for dark matter using antideuterons: the GAPS experiment. New J. Phys., 11(10):105022, 2009.

[7] Kaya Mori et al. A Novel antimatter detector based on x-ray deexcitation of exotic atoms. Astrophys. J., 566:604-616, 2002.

[8] C. J. Hailey et al. Antideuteron based dark matter search with GAPS: Current progress and future prospects. Adv. Space Res., 51:290-296, 2013.

[9] S. Quinn et al. The GAPS instrument: A large area time of flight and high resolution exotic atom spectrometer for cosmic antinuclei. This conference Proceedings of Science, 2021.

[10] S. Quinn. Recent Progress on the GAPS Time of Flight System. PoS, ICRC2019:128, 2019.

[11] Field Rogers et al. Large-area Si(Li) Detectors for X-ray Spectrometry and Particle Tracking for the GAPS Experiment. In 2019 IEEE Nuclear Science Symposium (NSS) and Medical Imaging Conference (MIC), 102019.

[12] N. Saffold et al. Passivation of $\mathrm{Si}(\mathrm{Li})$ detectors operated above cryogenic temperatures for space-based applications. Nucl. Instrum. Meth. A, 997:165015, 2021.

[13] M. Kozai et al. Developing a mass-production model of large-area $\mathrm{Si}(\mathrm{Li})$ detectors with high operating temperatures. Nucl. Instrum. Meth. A, 947:2695, 2019.

[14] V. Scotti et al. Front-end Electronics for the GAPS Tracker. PoS, ICRC2019:136, 2019.

[15] R. Munini et al. The antinucleus annihilation reconstruction algorithm of the GAPS experiment. Submitted on Astroparticle Physics, 2021.

[16] A. Tiberio et al. Reconstruction of antinucleus-annihilation events in the GAPS experiment. This conference Proceedings of Science, 2021. 
[17] S. Agostinelli et al. Geant4-a simulation toolkit. Nuclear Instruments and Methods in Physics Research Section A: Accelerators, Spectrometers, Detectors and Associated Equipment, 506(3):250-303, 2003.

[18] François Chollet. Deep Learning with Python. Manning, November 2017.

[19] S. Hochreiter et al. Long short-term memory. Neural computation, 9:1735-80, 121997.

[20] T. Aramaki et al. Antideuteron sensitivity for the gaps experiment. Astroparticle Physics, 74:6-13, 2016.

[21] Ruth Pordes, Don Petravick, Bill Kramer, Doug Olson, Miron Livny, Alain Roy, Paul Avery, Kent Blackburn, Torre Wenaus, Frank Würthwein, Ian Foster, Rob Gardner, Mike Wilde, Alan Blatecky, John McGee, and Rob Quick. The open science grid. 78:012057, 2007.

[22] Igor Sfiligoi, Daniel C Bradley, Burt Holzman, Parag Mhashilkar, Sanjay Padhi, and Frank Wurthwein. The pilot way to grid resources using glideinwms. 2:428-432, 2009. 


\section{Full Authors List: GAPS Collaboration}

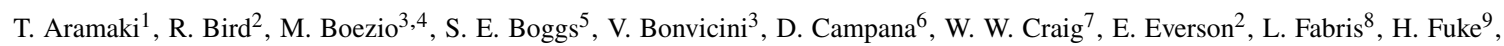
F. Gahbauer ${ }^{10}$, I. Garcia ${ }^{2}$, C. Gerrity ${ }^{11}$, C. J. Hailey ${ }^{10}$, T. Hayashi ${ }^{2}$, C. Kato ${ }^{12}$, A. Kawachi ${ }^{13}$, S. Kobayashi ${ }^{13}$, M. Kozai ${ }^{9}$, A. Lenni ${ }^{3,14}$, A. Lowell ${ }^{7}$, M. Manghisoni ${ }^{15,16}$, N. Marcelli ${ }^{17,18}$, B. Mochizuki ${ }^{7}$ S. A. I. Mognet ${ }^{19}$, K. Munakata ${ }^{12}$, R. Munini ${ }^{3,4}$, Y. Nakagami $^{20}$, J. Olson ${ }^{21}$, R. A. $\mathrm{Ong}^{2}$, G. Osteria ${ }^{6}$, K. Perez ${ }^{22}$, S. Quinn' ${ }^{2}$, V. Re ${ }^{15,16}$, E. Riceputi ${ }^{15,16}$, B. Roach ${ }^{22}$, F. Rogers ${ }^{22}$, J. A. Ryan ${ }^{2}$, N. Saffold ${ }^{10}$, V. Scotti ${ }^{6,23}$, Y. Shimizu ${ }^{24}$, M. Sonzogni ${ }^{15,16}$, R. Sparvoli ${ }^{17,18}$, A. Stoess $1^{11}$, A. Tiberio ${ }^{25}$, E. Vannuccini ${ }^{25}$, P. von Doetinchem $^{11}$, T. Wada ${ }^{20}$, M. Xiao 22 , M. Yamatami ${ }^{9}$, A. Yoshida ${ }^{20}$, T. Yoshida ${ }^{9}$, G. Zampa ${ }^{3}$, and J. Zweerink ${ }^{2}$

${ }^{1}$ Northeastern University, 360 Huntington Avenue, Boston, MA 02115, USA. ${ }^{2}$ University of California, Los Angeles, Los Angeles, CA 90095, USA. ${ }^{3}$ INFN, Sezione di Trieste, I-34149 Trieste, Italy. ${ }^{4}$ IFPU, I-34014 Trieste, Italy. ${ }^{5}$ University of California, San Diego, La Jolla, CA 90037, USA. ${ }^{6}$ INFN, Sezione di Napoli, I-80126 Naples, Italy. ${ }^{7}$ Space Sciences Laboratory, University of California, Berkeley, 7 Gauss Way, Berkeley, CA 94720, USA. ${ }^{8}$ Oak Ridge National Laboratory, Oak Ridge, TN 37831, USA. ${ }^{9}$ Institute of Space and Astronautical Science, Japan Aerospace Exploration Agency (ISAS/JAXA), Sagamihara, Kanagawa 252-5210, Japan. ${ }^{10}$ Columbia University, New York, NY 10027, USA. ${ }^{11}$ University of Hawaii at Manoa, Honolulu, HI 96822 USA. ${ }^{12}$ Shinshu University, Matsumoto, Nagano 390-8621, Japan. ${ }^{13}$ Tokai University, Hiratsuka, Kanagawa 259-1292, Japan. ${ }^{14}$ Universitá di Trieste, I-34127 Trieste, Italy. ${ }^{15}$ INFN, Sezione di Pavia, I-27100 Pavia, Italy. ${ }^{16}$ Universitá di Bergamo, I-24044 Dalmine (BG), Italy. ${ }^{17}$ INFN, Sezione di Rome "Tor Vergata", I-00133 Rome, Italy. ${ }^{18}$ Universitá di Roma "Tor Vergata", I-00133 Rome, Italy. ${ }^{19}$ Pennsylvania State University, University Park, PA 16802 USA. ${ }^{20}$ Aoyama Gakuin University, Sagamihara, Kanagawa 252-5258, Japan. ${ }^{21}$ Heliospace Corporation, Berkeley, CA 94710, USA. ${ }^{22}$ Massachusetts Institute of Technology, Cambridge, MA 02139, USA. ${ }^{23}$ Universitá di Napoli "Federico II", I-80138 Naples, Italy. ${ }^{24}$ Kanagawa University, Yokohama, Kanagawa 221-8686, Japan. ${ }^{25}$ INFN, Sezione di Firenze, I-50019 Sesto Fiorentino, Florence, Italy. 